\title{
Ubiquity of amplitude-modulated magnetic ordering in the $H-T$ phase diagram of the frustrated non-Fermi-liquid YbAgGe
}

\author{
C. B. Larsen $\odot,{ }^{1}$ E. Canévet, ${ }^{1}$ D. G. Mazzone, ${ }^{1}$ Ch. Rüegg,,${ }^{1,2,3}$ B. Fåk,${ }^{4}$ D. F. McMorrow,${ }^{3}$ E. Ressouche,${ }^{5}$ \\ G. J. McIntyre ${ }^{0},{ }^{4, *}$ S. L. Bud'ko, ${ }^{6}$ P. C. Canfield ${ }^{\circ},{ }^{6}$ and O. Zaharko ${ }^{1, \dagger}$ \\ ${ }^{1}$ Laboratory for Neutron Scattering and Imaging, Paul Scherrer Institute, CH-5232 Villigen, Switzerland \\ ${ }^{2}$ Department of Quantum Matter Physics, University of Geneva, CH-1211 Geneva, Switzerland \\ ${ }^{3}$ London Centre for Nanotechnology and Department of Physics and Astronomy, University College London, \\ London WC1E 6BT, United Kingdom \\ ${ }^{4}$ Institut Laue-Langevin, 71 Avenue des Martyrs, CS20156, 38042 Grenoble Cédex 9, France \\ ${ }^{5}$ University Grenoble Alpes, CEA, IRIG, MEM, MDN, 38000 Grenoble, France \\ ${ }^{6}$ Ames Laboratory and Department of Physics and Astronomy, Iowa State University, Ames, Iowa 50010, USA
}

(Received 9 June 2021; accepted 30 July 2021; published 17 August 2021)

\begin{abstract}
YbAgGe contains a magnetic geometrically frustrated kagome-like lattice that also features significant local single-ion anisotropy. The electronic state is established by hybridization of $4 f$ and conduction electrons leading to heavy electronic masses. The competition between these various interactions leads to nontrivial behavior under external magnetic field, including a sequence of magnetic phase transitions, non-Fermi-liquid states, and possibly a quantum critical point. We present a series of neutron diffraction experiments performed in the $\mathrm{mK}$ temperature range and under magnetic fields up to $8 \mathrm{~T}$ in the hexagonal plane, revealing the microscopic nature of the first four subsequent magnetic states of this phase diagram. The magnetic phases are associated with

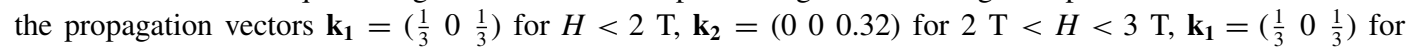
$3 \mathrm{~T}<H<4.5 \mathrm{~T}$, and $\mathbf{k}_{3}=(0.1950 .1950 .38)$ for $4.5 \mathrm{~T}<H<7 \mathrm{~T}$. Our structural refinements reveal a strong modulation of the magnetic moment amplitude in all phases. We observe that the ordered moments of the three magnetically different $\mathrm{Yb}$ sites become increasingly different in field, which complies with the principle local anisotropy directions relative to the field direction. While the ordered moments are aligned predominantly in the hexagonal plane, we also find a significant out-of-plane component and a ferromagnetic contribution above $2 \mathrm{~T}$. We discuss possible scenarios that may evolve around the phase boundary at $4.5 \mathrm{~T}$, which is associated with putative quantum criticality as identified by various bulk probes. We propose further steps that are required to better understand the microscopic interactions in this material.
\end{abstract}

DOI: 10.1103/PhysRevB.104.054424

\section{INTRODUCTION}

Several topical themes of modern solid-state researchfrustrated magnetism, non-Fermi-liquid behavior, and fieldinduced quantum criticality-converge in $\mathrm{YbAgGe}$, a system that has been in the focus of experimental studies for more than a decade [1-12].

YbAgGe belongs to a family of rare-earth (R) compounds with noncentrosymmetric crystal structure (ZrNiAl-type, space group $P \overline{6} 2 m)$, where the $\mathrm{R}^{3+}$-ions occupy the $3(f)$ Wyckoff position $\left(C_{2 v}\right.$ site symmetry) and form a twodimensional, distorted kagome-like lattice of corner-sharing equilateral triangles [13]. Some members of this family, i.e., TbPtIn and TmAgGe [14,15], possess a very strong easyaxis anisotropy in the hexagonal plane, which is imposed by the crystal-electric field (CEF). This leads to a susceptibility ratio of $\chi_{a b} / \chi_{c} \approx 30$ at $5 \mathrm{~K}$, and triggers a series of

\footnotetext{
*Present address: Australian Nuclear Science and Technology Organisation, Lucas Heights NSW 2234, Australia.

†oksana.zaharko@psi.ch
}

metamagnetic transitions in which the magnetic moments are restricted along the three distinct axes defined by the CEF, and which are selected by the magnetic field direction [15]. The sister compound HoAgGe features a kagome spin-ice state that is established through the combination of single-ion axial anisotropy in the hexagonal plane and effective ferromagnetic nearest-neighbor exchange interactions [16]. Under field this state evolves into ordered and partially disordered magnetic states, all obeying the kagome ice rule.

The magnetic moments in YbAgGe also show axial anisotropy within the hexagonal plane, but here they couple antiferromagnetically. The material features a Curie-Weiss temperature of $\theta_{C W}=-30 \mathrm{~K}$ and an anisotropy ratio that reaches moderate $\chi_{a b} / \chi_{c} \approx 3$ at low temperature [14]. Inelastic neutron scattering (INS) results suggest a doubly degenerate CEF ground state $\left|J=7 / 2, J_{z}= \pm 7 / 2\right\rangle$ [9]. The local quantization axis for the three magnetic $\mathrm{Yb}$ sites of the unit cell $[\mathrm{Yb} 1=(\mathrm{x}, 0,0), \mathrm{Yb} 2=(0, \mathrm{x}, 0), \mathrm{Yb} 3=(-\mathrm{x},-\mathrm{x}, 0)$, $\mathrm{x}=0.58$ ] points along the $z=\left[\begin{array}{lll}1 & 0 & 0\end{array}\right],\left[\begin{array}{lll}0 & 1 & 0\end{array}\right]$, or the [ [ $\left.\begin{array}{lll}1 & 1 & 0\end{array}\right]$ two-fold axes, respectively. Thus the anisotropy is essentially the same as in the TmAgGe [14] member of the RAgGe family as well as the isostructural TbPtIn [14]. 

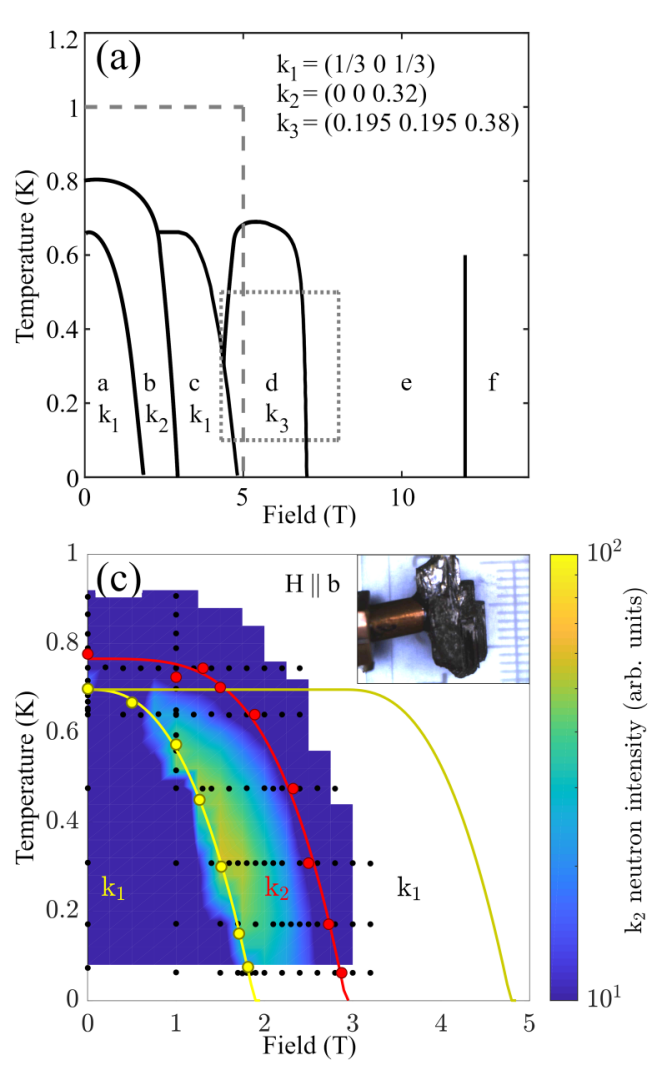
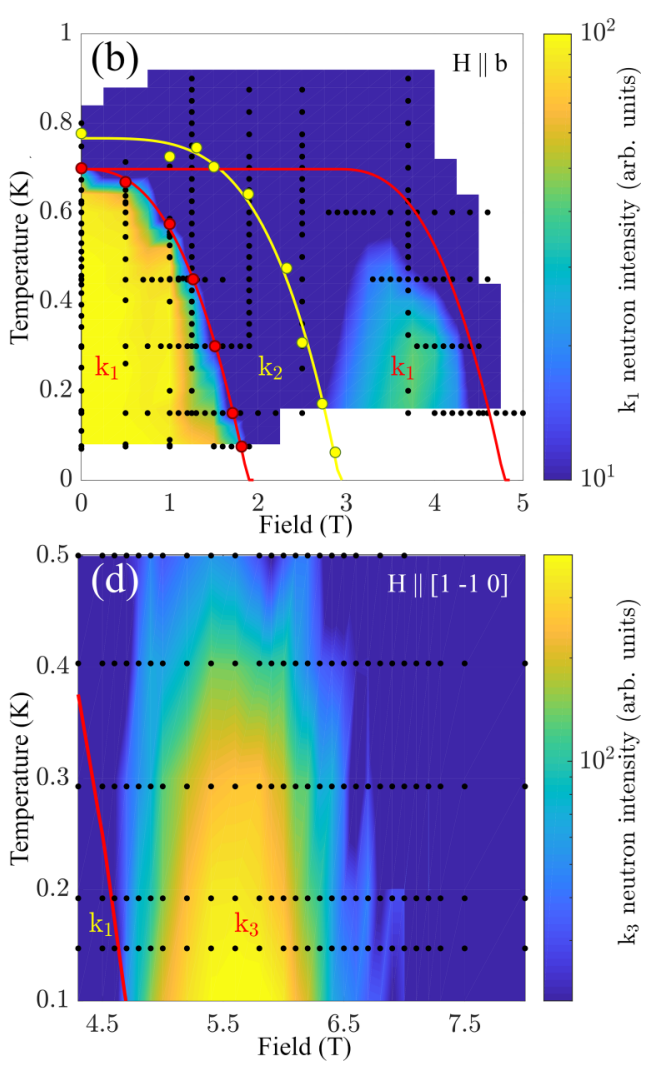

FIG. 1. (a) Schematic $H-T$ phase diagram of YbAgGe. Solid lines mark approximate phase boundaries. [(b), (c)] Maps of the magnetic intensity for selected reflections corresponding to $\mathbf{k}_{1}$ and $\mathbf{k}_{2}$ propagation vectors, respectively [see also dashed region in (a)]. (d) Map of the magnetic intensity for $\mathbf{k}_{3}$ in the dotted region of panel (a). The yellow and red lines indicate the approximate magnetic phase boundaries, while black dots indicate the exact measurement points. The magnetic field was applied along the $\hat{b}$ direction in (b) and (c) and along [1 -10$]$ in (d). The inset in (c) shows a $14 \times 8 \times 8 \mathrm{~mm}^{3} \mathrm{YbAgGe}$ single crystal on a copper mount.

Low-temperature thermodynamic and transport results $[1-5,14,17,18]$ distinguish $\mathrm{YbAgGe}$ from other RAgGe compounds with well localised $\mathrm{R}$ moments. The electronic specific-heat coefficient $\gamma$ ranges from 0.15 to $1 \mathrm{~J} / \mathrm{molK}^{2}$ and the entropy release is only $\sim 5 \%$ of $R \ln 2$ at $1 \mathrm{~K}$ $[1,3,14]$. This signifies that $\mathrm{YbAgGe}$ may be on the border of magnetic moment formation, which is established through hybridization between $4 f$ and conduction electrons. Specific-heat and resistivity measurements under magnetic field and $\mathrm{mK}$ temperatures suggest non-Fermi-liquid behavior and quantum-critical fluctuations at the field values of $H_{\text {crit }}^{a b} \approx$ $4.5 \mathrm{~T}$ and $H_{\text {crit }}^{c} \approx 8 \mathrm{~T}$ for field directions in the hexagonal plane and along the $\mathrm{c}$ axis, respectively $[1,3,5,7,12]$.

The complex $H-T$ phase diagram of YbAgGe [Fig. 1(a)], constructed from detailed bulk measurements $[1-5,14,17,18]$, contains up to six different phases labeled by the letters $a$ to $f$ [5]. Earlier magnetic neutron elastic-scattering studies were able to identify the ordering wave vectors of the first two of these phases. At zero field the magnetic $\mathrm{Yb}^{3+}$ moments order

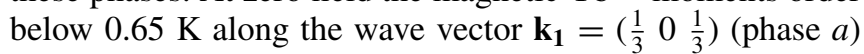
[8]. Phase $b$ features the incommensurate wave vector $\mathbf{k}_{\mathbf{2}}=(0$ 0 0.32) [10] and encapsulates the phase $a$ in the $H-T$ phase diagram. It extends up to $T_{N} \approx 0.8 \mathrm{~K}$ at zero field, after which magnetic order vanishes. At low temperatures $(T<0.65 \mathrm{~K})$, phase $b$ exists within $2 \mathrm{~T}<H<3 \mathrm{~T}$.

The magnetic order of the higher-field phases $c, d$, and $e$ have remained elusive to this date. However, the $c-d$ phase boundary has been intensively studied because of numerous anomalous properties. The Hall resistivity is enhanced [2], the sign of the Grüneisen parameter changes [7], the magnetization peaks [19], and the elastic constants soften [6]. Detailed magnetocaloric measurements [7] classify the sharp transition at $T_{B C P} \approx 0.3 \mathrm{~K}$ and $H_{B C P} \approx 4.5 \mathrm{~T}$ as a bicritical point (BCP), which may be tuned to become quantum critical.

Thus, the evolution of the field-induced magnetic structures in $\mathrm{YbAgGe}$, especially the ones for phases $c$ and $d$, is indispensable for our understanding of the complex interplay between the geometrical frustration of $\mathrm{Yb}^{3+}$ moments on the kagome-like lattice, their local orthorhombic CEF anisotropy, and their interaction with the conduction electrons. Here we report neutron diffraction experiments on $\mathrm{YbAgGe}$ single crystals in zero and applied magnetic fields. We identify the corresponding propagation vectors and determine the magnetic structures of all four successive states $a, b, c$, and $d$.

\section{EXPERIMENTAL DETAILS}

\section{A. Sample preparation}

YbAgGe single crystals were grown from Ag- and Ge-rich high-temperature ternary solutions and characterized based on the procedure described in Ref. [14]. In order to grow a significant number of large single crystals of $\mathrm{YbAgGe}$, the growth was optimized in several ways. 
Roughly $10 \mathrm{~g}$ of high-purity $\mathrm{Yb}, \mathrm{Ag}$, and $\mathrm{Ge}$ were placed in a $5 \mathrm{ml} \mathrm{Al} \mathrm{O}_{3}$ crucible in a molar ratio of $\mathrm{Yb}_{0.14} \mathrm{Ag}_{0.645} \mathrm{Ge}_{0.215}$. The $\mathrm{Yb}$ with $99.99+\%$ purity was obtained from the Ames Laboratory Materials Preparation Center. A second $5 \mathrm{ml}$ $\mathrm{Al}_{2} \mathrm{O}_{3}$ crucible, filled with silica wool, was placed on top of the growth crucible to act as a filter and catch for excess liquid [20]. The growth and catch crucibles were sealed into an evacuated amorphous silica tube and placed in a resistively heated box furnace. The furnace was heated to $1100{ }^{\circ} \mathrm{C}$ over 4 hours, and heated further to $1190^{\circ} \mathrm{C}$ over an additional hour. The furnace was then cooled to $1090^{\circ} \mathrm{C}$ over 5 hours and then slow cooled to $840{ }^{\circ} \mathrm{C}$ over 200 hours. The growth crucibles were removed from the furnace at $840{ }^{\circ} \mathrm{C}$ and placed in a centrifuge for separation of the excess liquid from the $\mathrm{YbAgGe}$ single crystals. Depending on nucleation, single crystals as large as 1-2 grams could be obtained [see inset to Fig. 1(c) for an example].

\section{B. Neutron diffraction setups}

A series of neutron-diffraction experiments was performed on the single-crystal neutron diffractometers D10 and D23 at the Institut Laue-Langevin (ILL), France, and on the singlecrystal neutron diffractometer Zebra (former TriCS) at the Swiss Neutron Spallation Source SINQ, Paul Scherrer Institut (PSI), Switzerland.

D10 was operated with a wavelength of $2.36 \AA$ in fourcircle mode with a dilution refrigerator at $140 \mathrm{mK}$ in zero magnetic field. The Zebra (TriCS) and D23 experiments used neutron wavelengths of $2.43 \AA$ and $2.366 \AA$, respectively. The last two diffractometers were operated with lifting-arm normal-beam geometry and were equipped with dilution refrigerators inserted into vertical magnets. For the study of the $H-T$ phase diagram, the $\mathrm{YbAgGe}$ crystals were mounted and cooled to a base temperature of $60 \mathrm{mK}$ on Zebra (TriCS) and D23. The field was applied in the hexagonal plane, either along [ $\left[\begin{array}{ll}1 & -1\end{array}\right]$ or along the $\hat{b}$ axis. According to bulk measurements $[3,9]$, the essential features of the phase diagram remain unchanged for these different field directions, but the phase boundaries may slightly shift. A consistent zero-field cooling protocol was used in all experiments, i.e., after each field ramp the crystal was heated to $1 \mathrm{~K}$ and cooled in zero field to base temperature. Data sets for the three field-induced phases were collected at magnetic fields of $2.2 \mathrm{~T}, 3.9 \mathrm{~T}$, and $5.5 \mathrm{~T}$ applied along $H_{1 \overline{1} 0}$. Our neutron-diffraction experiments reveal that magnetic reflections occurring in phases $a, b, c$, and $d$ [Fig. 1(a)] can be indexed with the propagation vectors $\mathbf{k}_{\mathbf{1}}$ [Fig. 1(b)], k $\mathbf{k}_{\mathbf{2}}$ [Fig. 1(c)], $\mathbf{k}_{\mathbf{1}}$ [Fig. 1(b)], and $\mathbf{k}_{\mathbf{3}}$ [Fig. 1(d)], respectively. The $a$ phase with propagation vector $\mathbf{k}_{\mathbf{1}}$ is separated from the other phases by a first-order transition, and shows a clear hysteresis. Meanwhile, the phase transition of phase $b$ is second order when approached from high temperature or field, but is first order when entered from the $a$-phase. We observed weak intensities at the $\mathbf{k}_{\mathbf{1}}$ positions that coexist with the $\mathbf{k}_{\mathbf{2}}$ reflections in the $b$ phase, suggesting a competition between these two magnetic states. Magnetic Bragg reflections at $\mathbf{k}_{\mathbf{1}}$ reappear in the $c$ phase, albeit with a different domain population and moment direction when compared to the zero-field state (see further below). The propagation vector $\mathbf{k}_{\mathbf{3}}$ of the $d$ phase was found by extensive reciprocal (hhl)-plane mapping.

We also searched for new propagation vectors in the $e$ phase by mapping the reciprocal $(\mathrm{h} 0 \mathrm{l})$ plane from $\left(\begin{array}{lll}0.25 & 0 & 0\end{array}\right)$ to $(0.8701)$ and the $(\mathrm{hhl})$ plane from $(0.10 .10)$ to $(0.70 .71)$ at $8 \mathrm{~T}$. However, no magnetic signal was found.

\section{Exploration of the phase diagram}

Figure 2 presents the field evolution of the antiferromagnetic reflections $\left(\frac{1}{3}-\frac{1}{3} \quad \frac{1}{3}\right),\left(\begin{array}{lll}1 & 0 & 0.32\end{array}\right)$, and (0.195 0.1950 .62$)$ alongside the magnetic contribution to the nuclear (110) Bragg peak. The different slopes of rising and decreasing intensities at various phase boundaries suggest possible crossovers from second- to first-order transitions, which is in agreement with magnetization, dilation and thermopower results $[4,5,14,17,19]$. The magnetic contribution to (110) sets in at the $b$ phase and continues to rise with a similar

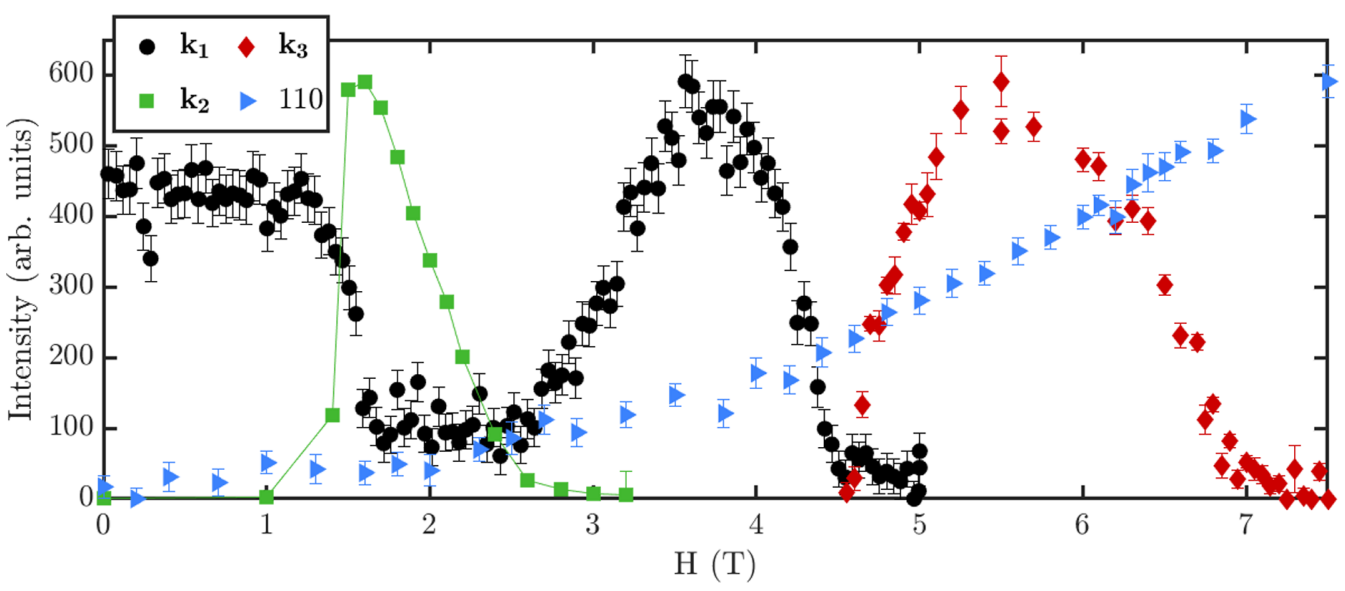

FIG. 2. Field dependence of the $\left(\frac{1}{3}-\frac{1}{3} \frac{1}{3}\right)\left(\mathbf{k}_{\mathbf{1}}\right)$ and (0.195 0.195 0.62) (k $\left.\mathbf{k}_{\mathbf{3}}\right)$ magnetic Bragg peaks overplotted with the nuclear (110) Bragg intensity, measured with increasing field $H_{[1 \overline{1} 0]}$ at base temperature. The plot also includes the field dependence of the magnetic (1 00.32$)$ ( $\mathbf{k}_{\mathbf{2}}$ ) Bragg peak measured with the field along the $\hat{b}$ axis. The intensities with different colours have been measured in different experiments and have been rescaled to the same maximum intensity. Thus they are not directly comparable. 
TABLE I. The arms of each propagation vector $\mathbf{k}$ ( $-\mathbf{k}$ arms are not listed explicitly) and the overview of the data sets measured at four magnetic fields. $\checkmark$ and - denote data sets with significant and negligible intensity, respectively. The number of reflections considered for the magnetic structure refinement is given in brackets.

\begin{tabular}{|c|c|c|c|c|c|}
\hline \multirow[b]{2}{*}{$\mathbf{k}$} & \multirow[b]{2}{*}{$\mathbf{k}^{\mathrm{arm}}$} & \multicolumn{4}{|c|}{$H(\mathrm{~T})$} \\
\hline & & 0 & 2.2 & 3.9 & 5.5 \\
\hline \multirow[t]{6}{*}{$\mathbf{k}_{1}$} & $\left(\frac{1}{3} 0 \frac{1}{3}\right)$ & $\checkmark$ & & - & \\
\hline & $\left(-\frac{1}{3} \frac{1}{3}-\frac{1}{3}\right)$ & $\checkmark$ & & $\checkmark(111)$ & \\
\hline & $\left(0 \frac{1}{3} \frac{1}{3}\right)$ & $\checkmark$ & & - & \\
\hline & $\left(0 \frac{1}{3}-\frac{1}{3}\right)$ & $\checkmark(127)$ & & - & \\
\hline & $\left(\frac{1}{3}-\frac{1}{3}-\frac{1}{3}\right)$ & $\checkmark$ & & $\checkmark$ & \\
\hline & $\left(\frac{1}{3} 0-\frac{1}{3}\right)$ & $\checkmark$ & & - & \\
\hline $\mathbf{k}_{2}$ & $\left(\begin{array}{lll}0 & 0 & 0.32\end{array}\right)$ & & $\checkmark(96)$ & & \\
\hline \multirow[t]{6}{*}{$\mathbf{k}_{3}$} & (0.195 0.1950 .380$)$ & & & & $\checkmark(92)$ \\
\hline & $(-0.3900 .195-0.380)$ & & & & - \\
\hline & ( $0.195-0.3900 .380)$ & & & & - \\
\hline & $(0.195-0.390-0.380)$ & & & & - \\
\hline & $(0.1950 .195-0.380)$ & & & & $\checkmark(92)$ \\
\hline & $\left(\begin{array}{llll}-0.390 & 0.195 & 0.380\end{array}\right)$ & & & & - \\
\hline
\end{tabular}

slope through the $c$ and $d$ phases, which indicates complex conical moment arrangements.

\section{Domain population and data sets}

Each of the identified propagation vectors $\mathbf{k}$ is related to other wave vectors by symmetry operations of the paramagnetic space group. This forms a unique set, which is called the star of the propagation vector. During our experiments we extensively inspected the arms of all propagation vectors and we summarize their presence in Table I.

The field dependence of the pair of reflections $\left(\begin{array}{lll}\frac{1}{3} & 0 & \frac{1}{3}\end{array}\right)$ and $\left(\frac{1}{3}-\frac{1}{3} \frac{1}{3}\right)$, belonging to different arms of $\mathbf{k}_{\mathbf{1}}$, is shown in Fig. 3 . The first reflection is present only in the $a$ phase, whereas the second peak appears in both the $a$ and $c$ phases. Thus, the phases $a$ and $c$ feature the same propagation vector $\mathbf{k}_{\mathbf{1}}$, but a different number of arms are populated. In zero field, all twelve arms are observed, while at 3.9 T only four arms with in-plane components directed along the applied field remain populated (Fig. 4). Similarly, only four out of twelve arms reveal finite intensity in the $d$ phase at $5.5 \mathrm{~T}$. Their in-plane components are orthogonal to the field (Table I, Fig. 4). These observations may be rationalized in terms of magnetic configuration domains, which give rise to magnetic reflections of different arms. Determination of the domain population is important for refining the magnetic moment amplitude of a magnetic state.

The zero-field data set (phase $a$ ) collected on D10 contained 581 magnetic reflections belonging to all twelve arms of $\mathbf{k}_{\mathbf{1}}=\left(\begin{array}{lll}\frac{1}{3} & 0 & \frac{1}{3}\end{array}\right)$. The intensity distribution unambiguously showed that all six configuration domains were equally populated. We used 127 reflections of the $\left(0 \frac{1}{3}-\frac{1}{3}\right)$ arm to determine the magnetic structure. At $H=2.2 \mathrm{~T}$ (phase $b$ ), 96 magnetic reflections with propagation vector $\mathbf{k}_{2}=\left(\begin{array}{lll}0 & 0 & 0.325\end{array}\right)$ were collected. This phase has only one configuration domain and
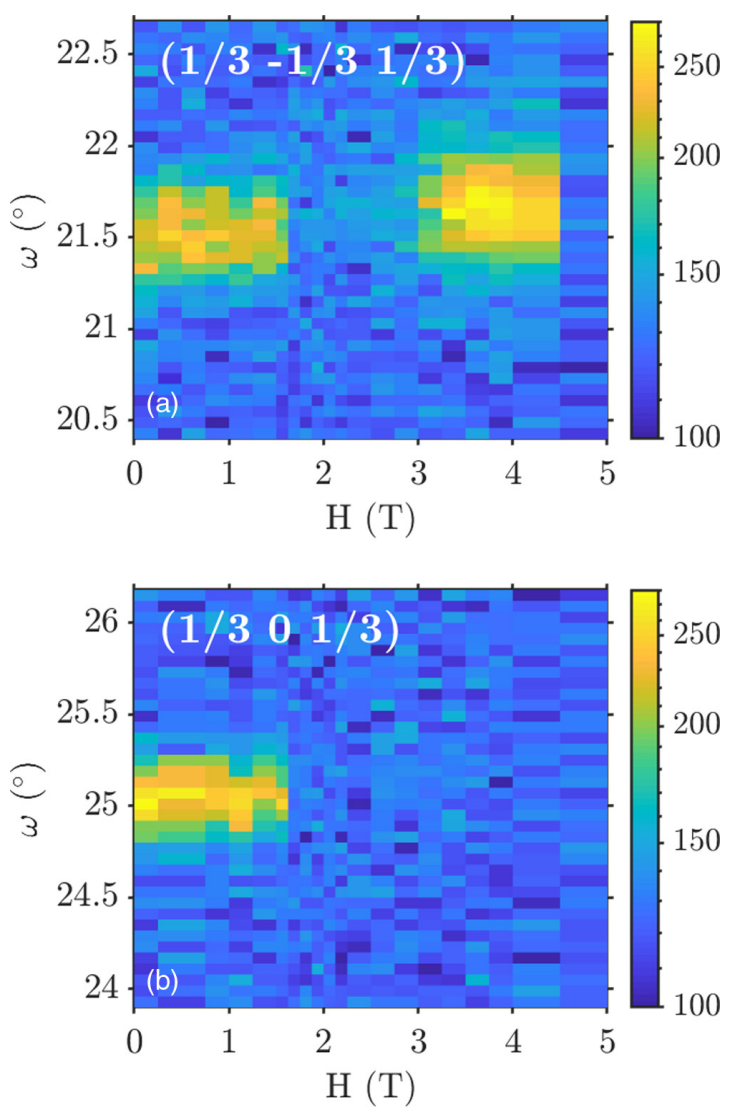

FIG. 3. Field evolution of the magnetic $\left(\begin{array}{lll}\frac{1}{3} & 0 & \frac{1}{3}\end{array}\right)$ and $\left(\begin{array}{lll}\frac{1}{3} & -\frac{1}{3} & \frac{1}{3}\end{array}\right)$ reflections for $H \|[1 \overline{1} 0]$. The angle $\omega$ is transversal to the scattering vector.

all reflections were used for the refinement of the magnetic structure. At $H=3.9 \mathrm{~T}$ (phase $c$ ), 443 reflections of the propagation vector $\mathbf{k}_{\mathbf{1}}$ were measured. We used 111 reflections of

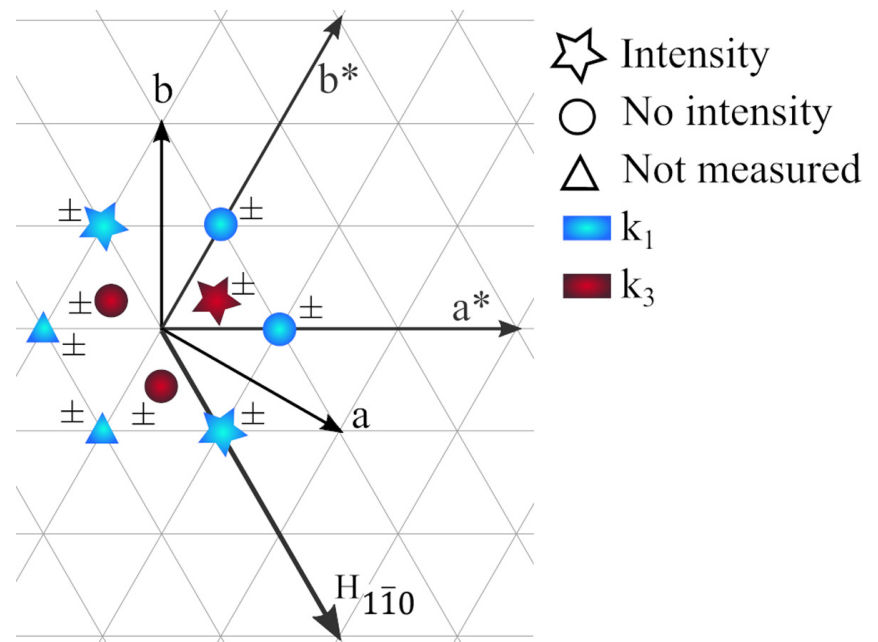

FIG. 4. Schematic view of the in-plane real-space lattice $(\hat{a} \hat{b})$, reciprocal-space lattice $\left(a^{*} b^{*}\right)$, and projection of the in-plane components of the $\mathbf{k}_{\mathbf{1}}$ and $\mathbf{k}_{\mathbf{3}}$ arms for $H \|[1 \overline{1} 0]$ at $3.9 \mathrm{~T}$ and $5.5 \mathrm{~T}$, respectively. Reflections with components above and below the hexagonal plane are indicated by \pm signs. Four of the $\mathbf{k}_{\mathbf{1}}$ arms could not be reached due to instrumental limitations and were therefore not measured. 
TABLE II. Overview of representation analysis of the three observed propagation vectors.

\begin{tabular}{lccc}
\hline \hline $\mathbf{k}$ & BZ point & IRR & \# Orbits \\
\hline $\mathbf{k}_{\mathbf{1}}$ & $\mathrm{D}(\alpha, 0, \gamma)$ & $\Gamma_{1}$ & 3 \\
& & $\Gamma_{2}$ & 2 \\
$\mathbf{k}_{\mathbf{2}}$ & $\Gamma_{1}$ & 1 \\
& & $\Gamma_{2}$ & 1 \\
& $\mathrm{DT}(0,0, \gamma)$ & $\Gamma_{3}$ & 1 \\
$\mathbf{k}_{3}$ & & $\Gamma_{1}$ & 1 \\
& $\mathrm{C}(\alpha, \alpha, \gamma)$ & $\Gamma_{2}$ & 1 \\
\hline \hline
\end{tabular}

the $\left(-\frac{1}{3} \frac{1}{3}-\frac{1}{3}\right)$ arm for the magnetic structure determination. At $H=5.5 \mathrm{~T}$ (phase $d$ ) 368 reflections for the star of $\mathbf{k}_{\mathbf{3}}=(0.195$ 0.1950 .380 ) were measured. Only 184 reflections belonging to two configuration domains had significant intensity and 92 reflections of the $(0.1950 .195-0.380)$ arm were used for further analysis.

\section{MAGNETIC STRUCTURE DETERMINATION}

\section{A. Magnetic symmetry and refinement strategy}

Symmetry representation analysis is a powerful method allowing to construct the magnetic structure models according to physically irreducible representations (IRRs) of the paramagnetic space group. We performed such analysis with the program Basireps [21] for $\mathbf{k}_{\mathbf{1}}, \mathbf{k}_{\mathbf{2}}$, and $\mathbf{k}_{\mathbf{3}}$. In all three cases, $\mathbf{k}$ and $\mathbf{- k}$ are not equivalent, and we considered the extended little group $\left(\mathbf{k}_{\mathbf{1}},-\mathbf{k}_{\mathbf{1}}\right)$. An overview of the basic symmetry properties of each solution is presented in Table II, while a more detailed description of the basis functions can be found in Table V. This approach is justified for second-order phase transitions and is comparable to considering only maximal subgroups. In the case of $\mathrm{YbAgGe}$, some transitions are of first order, and symmetry might therefore be lower, but we decided to stay within this approximation to reduce the number of refined parameters.

The wave vector $\mathbf{k}_{\mathbf{1}}=\left(\begin{array}{lll}\frac{1}{3} & 0 & \frac{1}{3}\end{array}\right)$ relates to the $\mathrm{D}$ point of the structural Brillouin Zone (BZ), $\mathrm{D}(\alpha, 0, \gamma)$. In our case the variables $\alpha$ and $\gamma$ attain the rational value $\frac{1}{3}$. None of the symmetry elements of the space group $P \overline{6} 2 m$ leave the wave vector $\mathbf{k}_{\mathbf{1}}$ invariant. The little group contains only the identity in standard representation analysis, and this leads to three independent $\mathrm{Yb}$ atoms. As we consider the extended little group $\left(\mathbf{k}_{\mathbf{1}},-\mathbf{k}_{\mathbf{1}}\right)$, two possible irreducible representations (IRRs) result. The first IRR, $\Gamma_{1}$, leaves the magnetic moments of the three $\mathrm{Yb}$ atoms in the unit cell independent. The second one, $\Gamma_{2}$, couples the moments of $\mathrm{Yb} 1$ and $\mathrm{Yb} 3$ (they form one orbit), while $\mathrm{Yb} 2$ remains independent.

The wave vector $\mathbf{k}_{\mathbf{2}}=\left(\begin{array}{lll}0 & 0 & 0.325\end{array}\right)$ belongs to the DT-point of the BZ, DT $(0,0, \gamma)$. Since we consider the extended little group $\left(\mathbf{k}_{\mathbf{2}},-\mathbf{k}_{\mathbf{2}}\right)$, the magnetic moments of the three $\mathrm{Yb}$ atoms are related by symmetry forming one orbit. Therefore three IRRs exist.

The wave vector $\mathbf{k}_{\mathbf{3}}=(0.1950 .1950 .38)$ is the $\mathrm{C}$ point of the $\mathrm{BZ}, \mathrm{C}(\alpha, \alpha, \gamma)$. In this case the symmetry analysis with the extended little group $\left(\mathbf{k}_{\mathbf{3}},-\mathbf{k}_{\mathbf{3}}\right)$ implies one orbit and two IRRs.
For each of the models, the modulated magnetic moment is represented as

$$
\begin{aligned}
\mathbf{m}_{l}(\mathbf{t})=\frac{1}{2} \sum_{j} & {\left[C_{j} \Psi_{j}^{l} \exp (-i 2 \pi \mathbf{k} \cdot \mathbf{t})\right.} \\
& \left.+\left(C_{j} \psi_{j}^{l}\right)^{*} \exp (i 2 \pi \mathbf{k} \cdot \mathbf{t})\right], \quad l=1,2,3,
\end{aligned}
$$

where $\Psi_{j}^{l}$ denotes the $j$ th basis function of moments at site $l$, $\mathbf{k}$ is the propagation vector, and $\mathbf{t}=\left(t_{a}, t_{b}, t_{c}\right)$ is a translation vector defining the unit cell. $C_{j}$ are refinement parameters that can be flagged as either real or imaginary, such that any model, including amplitude modulated ones defined by only real parameters or completely general helices defined by both real and imaginary parameters, can be considered.

To better understand the evolution of the magnetic moments with field, we introduce an orthogonal coordinate system $(\xi, \eta, \zeta)$ where $\xi$ is parallel to the magnetic field direction $[1,-1,0], \eta$ is along $[1,1,0]$, and $\zeta$ is along $[0,0,1]$. The moment on site $l=1 \ldots 3$ in unit cell $\mathbf{t}$ can then be written

$$
\mathbf{m}_{l}(\mathbf{t})=m_{l}^{H}(\mathbf{t}) \hat{\xi}+m_{l}^{[1,1,0]}(\mathbf{t}) \hat{\eta}+m_{l}^{c}(\mathbf{t}) \hat{\zeta},
$$

where $m_{l}^{H}$ is the moment component parallel to the applied field and $m_{l}^{\perp}(\mathbf{t})=\sqrt{\left[m_{l}^{[1,1,0]}(\mathbf{t})\right]^{2}+\left[m_{l}^{c}(\mathbf{t})\right]^{2}}$.

For each magnetic phase, we attempted refinements for all symmetry-allowed structures and for all possible permutations of real/imaginary $C_{j}$ flags. Parameters resulting from the best refinements for each IRR are listed in Table III, while the magnetic moment amplitudes of these structures are presented in Table IV. The determined magnetic structures are presented in Fig. 5.

\section{B. Zero-field $k_{1}$ phase}

The zero-field refinement results of Table III reveal that the best solutions for the $\Gamma_{1}$ and $\Gamma_{2}$ IRRs have similar goodnesses of fit and refinement parameters. This is likely a result of the high number of free parameters for both IRRs, which put only few restrictions on the magnetic moment. Therefore both IRRs are capable of approximating the experimental data well. Plots and further discussion of the zero-field model are based on parameters from the slightly better $\Gamma_{2}$ solution.

Figure 5(a) depicts a $\mathbf{t}=\left(t_{a}, t_{b}, 0\right)$ layer of the zero-field amplitude-modulated moment arrangement. The in-plane components of the $\mathrm{Yb} 1$ and $\mathrm{Yb} 2$ magnetic moments are aligned along the [lll $\left.\begin{array}{lll}1 & 0 & 0\end{array}\right]$ and [0 $\left[\begin{array}{ll}0 & 0\end{array}\right]$ directions, respectively, while the Yb3 moments are arranged along the diagonal $\left[\begin{array}{lll}1 & 1 & 0\end{array}\right]$. These orientations correspond to the principal directions of the CEF anisotropy due to the orthorhombic point symmetry of the 3(f) site [9]. The maximum moment value of $2.6(1) \mu_{B}$ is reduced compared to the free-ion value of $4.5 \mu_{B}$ for $\mathrm{Yb}^{3+}$. The average ordered moment amplitude, $\left\langle\left|\mathbf{m}_{l}(\mathbf{t})\right|\right\rangle$, is 1.87(4) $\mu_{B}$ for $\mathrm{Yb} 1$ and $\mathrm{Yb} 3$ and 1.04(4) $\mu_{B}$ for Yb2 (Table IV). Thus, the average moment associated with the amplitude modulated arrangement significantly exceeds the estimates of a static ordered moment of $0.1 \mu_{B}$ from the magnetic entropy calculations [3]. It might well be that the correlations probed in our diffraction experiment are not entirely elastic, but contain significant 
TABLE III. Refined basis-vector coefficients for selected arms. The $i$ th element of each entry in the "Flags" column refers to the designation of the corresponding refinement parameter $C_{i}$ as being either real or imaginary.

\begin{tabular}{|c|c|c|c|c|c|c|c|c|c|c|c|c|c|c|c|}
\hline IRR & $\mathbf{k}_{\text {meas }}$ & Flags & $C_{1}$ & $C_{2}$ & $C_{3}$ & $C_{4}$ & $\mathrm{C}_{5}$ & $C_{6}$ & $C_{7}$ & $C_{8}$ & $C_{9}$ & $\chi^{2}$ & $\mathrm{R}_{F}$ & $\mathrm{R}_{F^{2}}$ & $\mathrm{R}_{F^{2} w}$ \\
\hline \multicolumn{16}{|c|}{$\mathbf{k}_{\mathbf{1}}^{\mathrm{arm}}=\left(\begin{array}{lll}0 & 1 / 3-1 / 3\end{array}\right), H=0 \mathrm{~T}$} \\
\hline$\Gamma_{1}$ & & 011011100 & $-2.6(1)$ & $0.3(2)$ & $-1.1(3)$ & $1.05(7)$ & $0.28(7)$ & $0.5(1)$ & $-1.7(1)$ & $-0.7(2)$ & $0.2(2)$ & 31.8 & 14.7 & 24.2 & 26.8 \\
\hline \multicolumn{16}{|c|}{$\mathbf{k}_{\mathbf{2}}=\left(\begin{array}{lll}0 & 0 & 0.32\end{array}\right), H=2 \mathrm{~T}$} \\
\hline$\Gamma_{1}$ & & 0 & $-1.5(1)$ & & & & & & & & & 73.9 & 36.7 & 59.0 & 68.0 \\
\hline \multicolumn{16}{|c|}{$\mathbf{k}_{\mathbf{1}}^{\mathrm{arm}}=(1 / 3-1 / 31 / 3), H=3.9 \mathrm{~T}$} \\
\hline$\Gamma_{1}$ & & 111100111 & $-1.3(1)$ & $-1.8(1)$ & $-0.1(1)$ & $0.11(6)$ & $-0.88(5)$ & $0.21(6)$ & $0.6(1)$ & $0.17(8)$ & $-0.8(1)$ & 4.16 & 11.1 & 19.8 & 17.5 \\
\hline$\Gamma_{1}$ Fix ani & & 001101001 & $0.64(6)$ & 0 & $0.16(2)$ & $1.00(4)$ & $0.57(7)$ & $0.16(8)$ & $0.5(1)$ & 0 & $0.3(1)$ & 4.88 & 11.5 & 20.9 & 19.2 \\
\hline$\Gamma_{2}$ & & 111100110 & $0.92(9)$ & $1.2(1)$ & $-0.5(1)$ & $-0.4(1)$ & $1.26(7)$ & $1.4(1)$ & $-0.2(1)$ & $-0.12(5)$ & $0.4(1)$ & 4.19 & 11.1 & 19.6 & 17.6 \\
\hline$\Gamma_{2}$ Fix ani & & 000010101 & $-1.0(1)$ & 0 & $-0.5(2)$ & $-0.8(1)$ & $1.17(9)$ & 0 & $-0.4(1)$ & 0 & $-0.3(2)$ & 5.14 & 12.1 & 22.0 & 19.8 \\
\hline
\end{tabular}

inelastic components with energy lower than the energy of incoming neutrons (which for the wavelength of $2.36 \AA$ is $14.68 \mathrm{meV}$ ). Moment values exceeding those expected from entropy calculations have also been observed with Mössbauer spectroscopy, where a maximum moment value of $\approx 1.5 \mu_{B}$ based on a simple magnetic model has been estimated [11]. Tentative estimates from earlier neutron elastic scattering investigations also indicate moment values beyond the entropy prediction [10].

\section{C. $2.2 \mathrm{~T} \mathrm{k}_{2}$ phase}

For the $\mathbf{k}_{\mathbf{2}}$ state at $H=2.2 \mathrm{~T}$, the best goodness of fit is obtained for the $\Gamma_{3}$ magnetic structure. The Yb3 moments

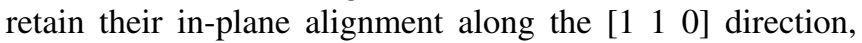
while the $\mathrm{Yb} 1$ and $\mathrm{Yb} 2$ moments swap directions such that the $\mathrm{Yb} 1$ moment is aligned along $\left[\begin{array}{lll}0 & 1 & 0\end{array}\right]$ and $\mathrm{Yb} 2$ along
[1 100$]$, hence breaking the CEF site-specific anisotropy. Attempting to force the moments to be aligned with their respective CEF anisotropy axes triples the $\chi^{2}$ value, indicating that the proposed solution is preferred. As evidenced from Table III, the goodness of fit of the optimal fit is noticeably worse than any of the solutions for the other phases. This suggests that lower symmetry solutions should be tried with an improved data set.

The absent in-plane components of the $\mathbf{k}_{\mathbf{2}}$ propagation vector ensure that there is no amplitude modulation of the magnetic moments in the hexagonal plane, while the amplitude modulation along the $\hat{c}$-axis is maintained. The in-plane components of the $\mathrm{Yb} 1, \mathrm{Yb} 2$, and $\mathrm{Yb} 3$ individual sublattices are collinear and the canting between the different sublattices is $60 / 120^{\circ}$. The average moment amplitudes of $\mathrm{Yb} 1, \mathrm{Yb} 2$, and Yb3 are $0.8(1), 1.2(1)$, and 1.0(1) $\mu_{B}$, thus lower than in the zero-field $\mathbf{k}_{\mathbf{1}}$ phase.

TABLE IV. Calculated magnetic quantities in $\left(\mu_{B}\right)$ : Maximum moment components along the three crystal axes $\hat{a}, \hat{b}$, and $\hat{c}$ for each $\mathrm{Yb}_{l}(1=1,2,3)$, maximum moment amplitude $m_{i, \max }$, average $m_{a v}$ and site-specific average $m_{i, a v}$ moment amplitudes.

\begin{tabular}{|c|c|c|c|c|c|c|c|c|c|c|}
\hline \multicolumn{11}{|c|}{$\mathbf{k}_{\mathbf{1}}=\left(\begin{array}{lll}1 / 3 & 0 & 1 / 3\end{array}\right), H=0 \mathrm{~T}$} \\
\hline$\Gamma_{1}$ & $(-2.59(1), 0.3(1),-1.0(2))$ & $(0.7(2),-1.8(1), 0.2(2))$ & $(-2.1(2),-2.1(2), 0.9(2))$ & $2.6(1)$ & $1.7(1)$ & $1.7(1)$ & $1.62(3)$ & $1.95(5)$ & $1.24(4)$ & $1.65(3)$ \\
\hline \multicolumn{11}{|c|}{$\mathbf{k}_{\mathbf{2}}=\left(\begin{array}{lll}0 & 0 & 0.32\end{array}\right), H=2.2 \mathrm{~T}$} \\
\hline$\Gamma_{3}$ & $(-0.1(2),-1.2(3), 0.2(2))$ & $(-1.5(2), 0.0(3), 0.63(9))$ & $(1.2(3), 1.5(3),-0.63(9))$ & $1.2(2)$ & $1.7(2)$ & $1.5(2)$ & $1.0(1)$ & $0.8(1)$ & $1.2(1)$ & $1.0(1)$ \\
\hline$\Gamma_{2}$ & $(-1.43(9),-1.7(1),-0.6(1))$ & $(0.10(5),-0.32(1), 0.4(1))$ & $(-1.43(9), 0.3(2), 0.3(1))$ & $1.7(1)$ & $0.42(8)$ & $1.64(9)$ & $0.93(2)$ & $1.25(3)$ & $0.40(3)$ & $1.14(3)$ \\
\hline \multicolumn{11}{|c|}{$\mathbf{k}_{\mathbf{1}}=(1 / 301 / 3), H=3.9 \mathrm{~T}, \mathrm{CEF}$ constrained } \\
\hline$\Gamma_{1}$ & $(0.64(6), 0,0.1(1))$ & $(0,0.5(1), 0.2(1))$ & $(-2.3(1),-1.74(7), 0.3(1))$ & $0.64(6)$ & $0.5(1)$ & $2.1(1)$ & $0.81(1)$ & $0.45(2)$ & $0.38(3)$ & $1.60(3)$ \\
\hline$\Gamma_{2}$ & $(1.49(9), 0,0.6(1))$ & $(0,-0.8(1),-0.3(1))$ & $(-1.49(9),-1.49(9),-0.6(1))$ & $1.50(9)$ & $0.8(1)$ & $1.50(9)$ & $0.94(2)$ & $1.12(3)$ & $0.61(3)$ & $1.12(4)$ \\
\hline \multicolumn{11}{|c|}{$\mathbf{k}_{\mathbf{3}}=\left(\begin{array}{lll}0.195 & 0.1950 .38\end{array}\right), H=5.5 \mathrm{~T}$} \\
\hline
\end{tabular}


TABLE V. Complex basis functions of the physically irreducible representations for the three propagation vectors $\mathbf{k}_{\mathbf{1}}=\left(\frac{1}{3} 0 \frac{1}{3}\right), \mathbf{k}_{\mathbf{2}}=\left(\begin{array}{lll}0 & 0 & 0.32\end{array}\right)$, and $\mathbf{k}_{\mathbf{3}}=(0.195,0.195,0.380)$.

\begin{tabular}{|c|c|c|c|c|c|}
\hline \multicolumn{2}{|c|}{ IRR BV } & $\begin{array}{c}\text { Yb1 } \\
(0.58,0,0)\end{array}$ & $\begin{array}{c}\mathrm{Yb} 2 \\
(0,0.58,0)\end{array}$ & \multicolumn{2}{|c|}{$\begin{array}{c}\text { Yb3 } \\
(0.42,0.42,0)\end{array}$} \\
\hline \multicolumn{6}{|c|}{$\mathbf{k}_{1}=\left(\begin{array}{lll}1 / 3 & 0 & 1 / 3\end{array}\right)$} \\
\hline & & $\mathrm{Re}$ & $\operatorname{Re}$ & $\operatorname{Re}$ & $\operatorname{Im}$ \\
\hline \multirow[t]{9}{*}{$\Gamma_{2}$} & $\Psi_{1}$ & $(\mathbf{1 . 0 0}, 0.00,0.00)$ & $(0.00,0.00,0.00)$ & $(\mathbf{0 . 5 0}, \mathbf{0 . 5 0}, 0.00)$ & $(\mathbf{0 . 8 7}, \mathbf{0 . 8 7}, 0.00)$ \\
\hline & $\Psi_{2}$ & $(0.00,1.00,0.00)$ & $(0.00,0.00,0.00)$ & $(0.00,-\mathbf{0 . 5 0}, 0.00)$ & $(0.00,-0.87,0.00)$ \\
\hline & $\Psi_{3}$ & $(0.00,0.00,1.00)$ & $(0.00,0.00,0.00)$ & $(0.00,0.00, \mathbf{0 . 5 0})$ & $(0.00,0.00, \mathbf{0 . 8 7})$ \\
\hline & $\Psi_{4}$ & $(0.00,0.00,0.00)$ & $(0.00,1.00,0.00)$ & $(0.00,0.00,0.00)$ & $(0.00,0.00,0.00)$ \\
\hline & $\Psi_{5}$ & $(\mathbf{1 . 0 0}, 0.00,0.00)$ & $(0.00,0.00,0.00)$ & $(-\mathbf{0 . 5 0},-\mathbf{0 . 5 0}, 0.00)$ & $(-0.87,-0.87,0.00)$ \\
\hline & $\Psi_{6}$ & $(0.00,1.00,0.00)$ & $(0.00,0.00,0.00)$ & $(0.00, \mathbf{0 . 5 0}, 0.00)$ & $(0.00, \mathbf{0 . 8 7}, 0.00)$ \\
\hline & $\Psi_{7}$ & $(0.00,0.00,1.00)$ & $(0.00,0.00,0.00)$ & $(0.00,0.00,-\mathbf{0 . 5 0})$ & $(0.00,0.00,-0.87)$ \\
\hline & $\Psi_{8}$ & $(0.00,0.00,0.00)$ & $(-1.00,-0.50,0.00)$ & $(0.00,0.00,0.00)$ & $(0.00,0.00,0.00)$ \\
\hline & $\Psi_{9}$ & $(0.00,0.00,0.00)$ & $(0.00,0.00,1.00)$ & $(0.00,0.00,0.00)$ & $(0.00,0.00,0.00)$ \\
\hline \multirow{9}{*}{$\Gamma_{1}$} & $\Psi_{1}$ & $(\mathbf{1 . 0 0}, 0.00,0.00)$ & $(0.00,0.00,0.00)$ & $(0.00,0.00,0.00)$ & $(0.00,0.00,0.00)$ \\
\hline & $\Psi_{2}$ & $(0.00,1.00,0.00)$ & $(0.00,0.00,0.00)$ & $(0.00,0.00,0.00)$ & $(0.00,0.00,0.00)$ \\
\hline & $\Psi_{3}$ & $(0.00,0.00,1.00)$ & $(0.00,0.00,0.00)$ & $(0.00,0.00,0.00)$ & $(0.00,0.00,0.00)$ \\
\hline & $\Psi_{4}$ & $(0.00,0.00,0.00)$ & $(0.00,0.00,0.00)$ & $(1.00,1.00,0.00)$ & $(1.73,1.73,0.00)$ \\
\hline & $\Psi_{5}$ & $(0.00,0.00,0.00)$ & $(0.00,0.00,0.00)$ & $(-1.00,0.00,0.00)$ & $(-1.73,0.00,0.00)$ \\
\hline & $\Psi_{6}$ & $(0.00,0.00,0.00)$ & $(0.00,0.00,0.00)$ & $(0.00,0.00,-1.00)$ & $(0.00,0.00,-1.73)$ \\
\hline & $\Psi_{7}$ & $(0.00,0.00,0.00)$ & $(0.00,1.00,0.00)$ & $(0.00,0.00,0.00)$ & $(0.00,0.00,0.00)$ \\
\hline & $\Psi_{8}$ & $(0.00,0.00,0.00)$ & $(-1.00,-1.00,0.00)$ & $(0.00,0.00,0.00)$ & $(0.00,0.00,0.00)$ \\
\hline & $\Psi_{9}$ & $(0.00,0.00,0.00)$ & $(0.00,0.00,1.00)$ & $(0.00,0.00,0.00)$ & $(0.00,0.00,0.00)$ \\
\hline \multicolumn{6}{|c|}{$\mathbf{k}_{2}=\left(\begin{array}{lll}0 & 0 & 0.32\end{array}\right)$} \\
\hline$\Gamma_{1}$ & $\Psi_{1}$ & $(\mathbf{0 . 5 0}, \mathbf{1 . 0 0}, 0.00)$ & $(-1.00,-0.50,0.00)$ & $(\mathbf{0 . 5 0},-\mathbf{0 . 5 0}, 0.00)$ & $(0.00,0.00,0.00)$ \\
\hline \multirow[t]{2}{*}{$\Gamma_{2}$} & & $(\mathbf{1 . 0 0}, 0.00,0.00)$ & $(0.00,1.00,0.00)$ & $(-1.00,-1.00,0.00)$ & $(0.00,0.00,0.00)$ \\
\hline & & $(0.00,0.00,1.00)$ & $(0.00,0.00,1.00)$ & $(0.00,0.00, \mathbf{1 . 0 0})$ & $(0.00,0.00,0.00)$ \\
\hline \multirow[t]{6}{*}{$\Gamma_{3}$} & & $(\mathbf{1 . 0 0}, 0.00,0.00)$ & $(0.00,-0.50,0.00)$ & $(\mathbf{0 . 5 0}, \mathbf{0 . 5 0}, 0.00)$ & $(0.00,0.00,0.00)$ \\
\hline & & $(0.00,0.00,0.00)$ & $(-1.00,-0.50,0.00)$ & $(-0.50,0.50,0.00)$ & $(0.00,0.00,0.00)$ \\
\hline & & $(0.00,0.00,0.00)$ & $(0.00,0.00,1.00)$ & $(0.00,0.00,-1.00)$ & $(0.00,0.00,0.00)$ \\
\hline & & $(0.00,0.00,0.00)$ & $(0.00,-1.00,0.00)$ & $(-1.00,-1.00,0.00)$ & $(0.00,0.00,0.00)$ \\
\hline & & $(\mathbf{0 . 5 0}, \mathbf{1 . 0 0}, 0.00)$ & $(\mathbf{0 . 5 0}, \mathbf{0 . 2 5}, 0.00)$ & $(-0.25,0.25,0.00)$ & $(0.00,0.00,0.00)$ \\
\hline & $\Psi_{6}$ & $(0.00,0.00,1.00)$ & $(0.00,0.00,-0.50)$ & $(0.00,0.00,-0.50)$ & $(0.00,0.00,0.00)$ \\
\hline \multicolumn{6}{|c|}{$\mathbf{k}_{3}=\left(\begin{array}{lll}0.195 & 0.195 & 0.38\end{array}\right)$} \\
\hline \multirow[t]{7}{*}{$\Gamma_{1}$} & $\Psi_{1}$ & $(\mathbf{1 . 0 0}, 0.00,0.00)$ & $(0.00,-1.00,0.00)$ & $(0.00,0.00,0.00)$ & $(0.00,0.00,0.00)$ \\
\hline & & $(0.00,1.00,0.00)$ & $(-1.00,0.00,0.00)$ & $(0.00,0.00,0.00)$ & $(0.00,0.00,0.00)$ \\
\hline & & $(0.00,0.00,1.00)$ & $(0.00,0.00,-1.00)$ & $(0.00,0.00,0.00)$ & $(0.00,0.00,0.00)$ \\
\hline & & $(0.00,0.00,0.00)$ & $(0.00,0.00,0.00)$ & $(1.00,-1.00,0.00)$ & $(0.00,0.00,0.00)$ \\
\hline & $\Psi_{5}$ & $(0.00,0.00,0.00)$ & $(0.00,1.00,0.00)$ & $(1.00,1.00,0.00)$ & $(0.00,0.00,0.00)$ \\
\hline & & $(0.00,0.00,0.00)$ & $(-1.00,-1.00,0.00)$ & $(0.00,-1.00,0.00)$ & $(0.00,0.00,0.00)$ \\
\hline & & $(0.00,0.00,0.00)$ & $(0.00,0.00,1.00)$ & $(0.00,0.00,-1.00)$ & $(0.00,0.00,0.00)$ \\
\hline \multirow[t]{8}{*}{$\Gamma_{2}$} & & $(\mathbf{1 . 0 0}, 0.00,0.00)$ & $(0.00,1.00,0.00)$ & $(0.00,0.00,0.00)$ & $(0.00,0.00,0.00)$ \\
\hline & & $(0.00,1.00,0.00)$ & $(\mathbf{1 . 0 0}, 0.00,0.00)$ & $(0.00,0.00,0.00)$ & $(0.00,0.00,0.00)$ \\
\hline & & $(0.00,0.00,1.00)$ & $(0.00,0.00,1.00)$ & $(0.00,0.00,0.00)$ & $(0.00,0.00,0.00)$ \\
\hline & & $(0.00,0.00,0.00)$ & $(0.00,0.00,0.00)$ & $(-1.00,-1.00,0.00)$ & $(0.00,0.00,0.00)$ \\
\hline & & $(0.00,0.00,0.00)$ & $(0.00,0.00,0.00)$ & $(0.00,0.00, \mathbf{1 . 0 0})$ & $(0.00,0.00,0.00)$ \\
\hline & & $(0.00,0.00,0.00)$ & $(0.00,1.00,0.00)$ & $(-1.00,-1.00,0.00)$ & $(0.00,0.00,0.00)$ \\
\hline & $\Psi_{7}$ & $(0.00,0.00,0.00)$ & $(-1.00,-1.00,0.00)$ & $(0.00, \mathbf{1 . 0 0}, 0.00)$ & $(0.00,0.00,0.00)$ \\
\hline & $\Psi_{8}$ & $(0.00,0.00,0.00)$ & $(0.00,0.00,1.00)$ & $(0.00,0.00, \mathbf{1 . 0 0})$ & $(0.00,0.00,0.00)$ \\
\hline
\end{tabular}

\section{3.9 $\mathrm{T}$ phase with re-entrant $\mathrm{k}_{1}$ propagation vector}

The $\mathbf{k}_{\mathbf{1}}$ propagation vector re-enters at magnetic fields between 3 and 4.8 T. Nonconstrained refinements with both the $\Gamma_{1}$ and $\Gamma_{2}$ IRRs yield solutions with moments deviating from the CEF anisotropy axes. Forcing the Yb1 and Yb2 moments to be along their respective anisotropy axes results in slightly worse goodnesses of fit and we thus present all four solutions in Table III. Overall, the $\Gamma_{1}$ IRR results in slightly better goodnesses of fit for both the CEF-constrained and nonconstrained refinements compared to the $\Gamma_{2}$ refinements. Both solutions 

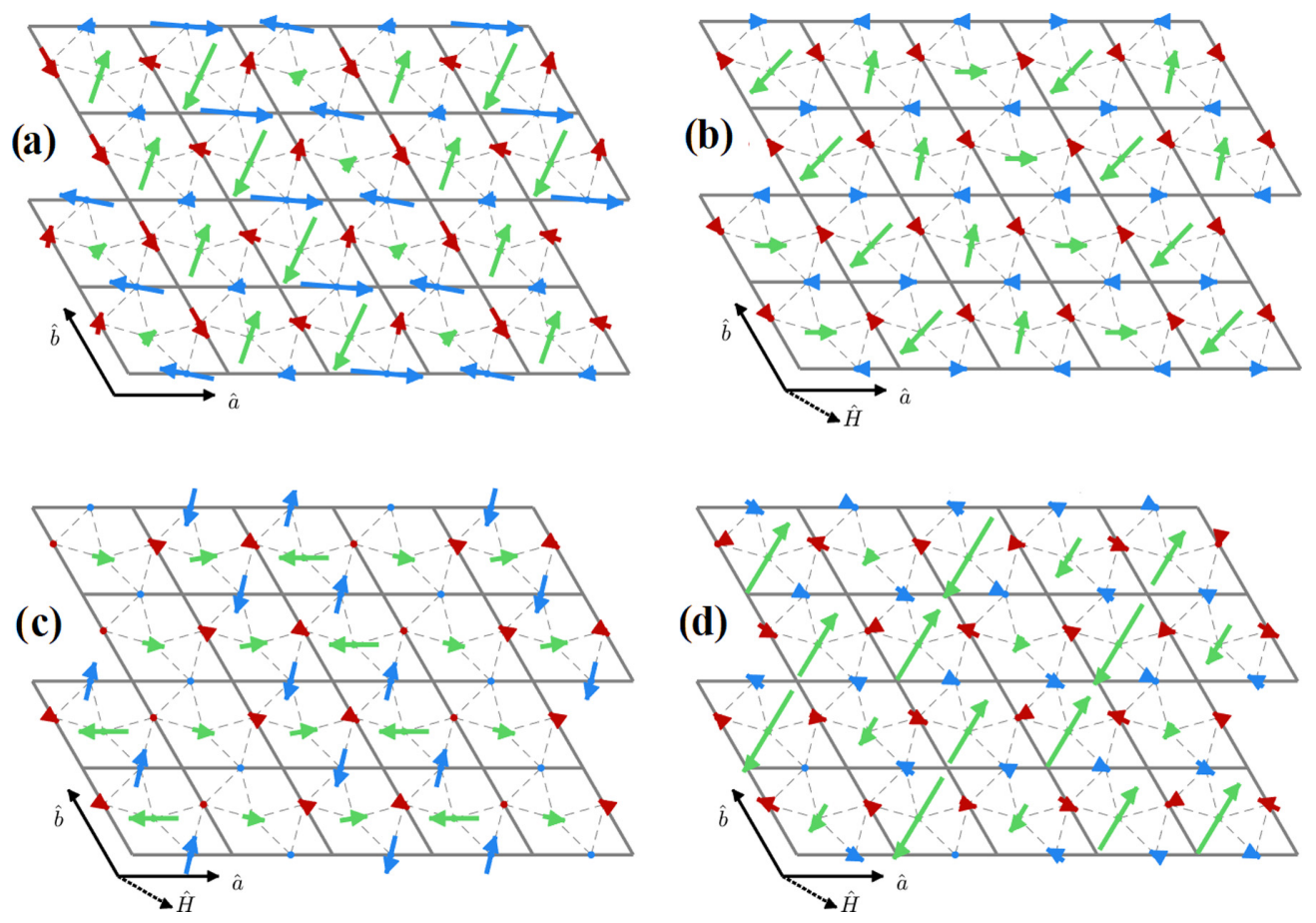

FIG. 5. A $\left(t_{x}, t_{y}, 0\right)$ layer of the magnetic structures for (a) $0-\mathrm{T} \Gamma_{2}$ solution with $\mathbf{k}_{\mathbf{1}}=\left(\frac{1}{3} 0 \frac{1}{3}\right)$, (b) 3.9-T $\Gamma_{3}$ solution with $\mathbf{k}_{\mathbf{1}}=\left(\frac{1}{3} 0 \frac{1}{3}\right)$ and enforced CEF anisotropy for Yb1 and Yb2 moments, (c) 3.9-T unconstrained $\Gamma_{1}$ solution with $\mathbf{k}_{1}=\left(\frac{1}{3} 0 \frac{1}{3}\right)$, and (d) 5.5-T $\Gamma_{2}$ solution with $\mathbf{k}_{\mathbf{3}}=(0.195$ 0.195 0.38). The magnetic moments of $\mathrm{Yb} 1, \mathrm{Yb} 2$, and $\mathrm{Yb} 3$ are coloured blue, red, and green, respectively. The magnetic field direction is $\left[\begin{array}{lll}1-1 & 0\end{array}\right]$ in (b), (c), and (d).

are shown in Figs. 5(b) and 5(c), respectively. For both $\Gamma_{1}$ solutions an unequal distribution of moment amplitudes is obtained. The average values are presented in Table IV. The unconstrained solution strongly breaks the CEF anisotropy as seen by the $\mathrm{Yb} 1$ and $\mathrm{Yb} 3$ moments being oriented primarily along [lll 110$]$ and [ $\left[\begin{array}{lll}1 & 0 & 0\end{array}\right]$, respectively. There is, thus, a large variance in the proposed magnetic structures, which all possess similar goodnesses of fit. This results in a certain ambiguity in the magnetic arrangement of the $c$ phase.

Both the constrained and unconstrained solutions exhibit decreased antiferromagnetic contributions, as gauged from the average magnetic-moment amplitudes of $0.81(1) \mu_{B}$ and 0.90 (1) $\mu_{B}$ compared to the zero-field solution with an average antiferromagnetic moment of 1.62(3) $\mu_{B}$. The decrease in the antiferromagnetic contribution may be compensated by an increasing ferromagnetic contribution in the $c$ phase as shown in Figs. 2 and 6.

\section{E. High-field $k_{3}$ phase}

The $d$-phase propagation vector $\mathbf{k}_{\mathbf{3}}=\left(\begin{array}{lll}0.195 & 0.1950 .38\end{array}\right)$ is observed at $5.5 \mathrm{~T}$, above the suggested bicritical point at $H_{\text {crit }}^{a b} \approx 4.5 \mathrm{~T}$. The best model, corresponding to the $\Gamma_{2}$ IRR, results in an amplitude-modulated structure with the in-plane components of the $\mathrm{Yb} 1, \mathrm{Yb} 2$, and $\mathrm{Yb} 3$ magnetic moments directed along their CEF anisotropy axes. In this structure, the average increase of magnetic moments is small when compared to the 3.9-T $\mathbf{k}_{\mathbf{1}}$ phase, but a stronger difference between the $\mathrm{Yb} 3$ moment and the $\mathrm{Yb} 1$ and $\mathrm{Yb} 2$ moments is apparent. The Yb3 moments reach on average 2.12(3) $\mu_{B}$, while the remaining $2 / 3$ of the moments are less than $1 \mu_{B}$, i.e., on average $0.51(1) \mu_{B} / \mathrm{Yb} 1$ and $0.66(1) \mu_{B} / \mathrm{Yb} 2$. This difference might be partly compensated by the ferromagnetic component, which is significant. However, its site variation has not been addressed yet.

\section{DISCUSSION}

We presented results of a series of neutron diffraction experiments on $\mathrm{YbAgGe}$ single crystals performed in the $\mathrm{mK}$ regime in zero and under magnetic fields applied in the hexagonal plane. We determined models of magnetic arrangements for the zero-field $a$ and the low-field $b$ phases with the previously reported $\mathbf{k}_{\mathbf{1}}$ and $\mathbf{k}_{\mathbf{2}}$ propagation vectors. We discovered the $\mathbf{k}_{\mathbf{1}}$ propagation vector in phase $c$ at $3.9 \mathrm{~T}$ and suggested the associated magnetic structure. The propagation vector in phase $d$ appearing above the critical field $H_{\text {crit }}^{a b} \approx 4.5 \mathrm{~T}$ is found to be $\mathbf{k}_{\mathbf{3}}=\left(\begin{array}{l}0.1950 .1950 .380\end{array}\right)$ and the corresponding magnetic arrangement is determined. The field-temperature extent of these four regions agrees very well with the $H-T$ phase diagrams established by extensive measurements of magnetization, specific heat, thermal expansion, magnetostriction, electrical resistivity, Hall effect, and thermoelectric power $[1-5,14,17,18]$.

Qualitatively, the low-field features of the magnetic phase diagram of $\mathrm{YbAgGe}$ (phases $a, b$, and $c$ ) follow the general trend for rare-earth systems. In zero-field cooling, the incommensurate magnetic $\mathbf{k}_{\mathbf{2}}$ order appears first. It locks into the commensurate $\mathbf{k}_{\mathbf{1}}$ state at low temperatures and then re-enters upon application of magnetic field. The mean-field model of Ref. [22] accounts for these features, assigning their origin to exchange interactions, while crystal electric fields impose 

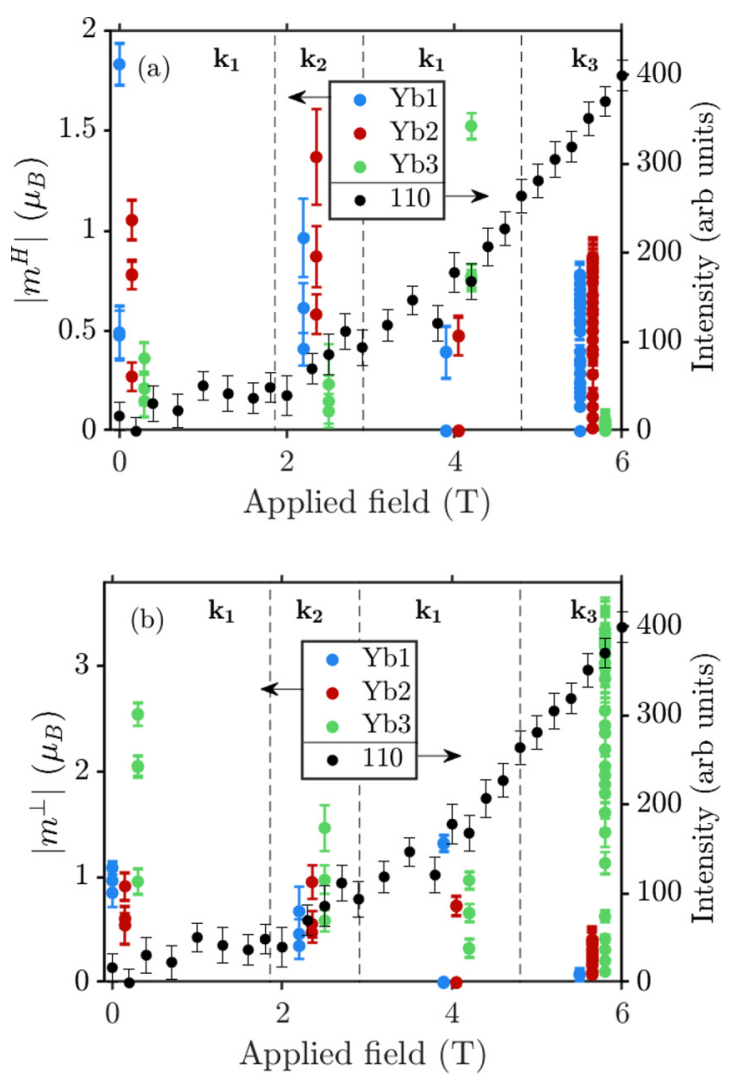

FIG. 6. Left scale: Parallel (a) and perpendicular (b) contribution of the magnetic moment with respect to the applied field $H_{[1-10]}$. All modulated moment amplitudes within the magnetic unit cell of the best refined models have been included, explaining the multiple values reported for $\mathrm{Yb} 1, \mathrm{Yb} 2$, and $\mathrm{Yb} 3$ at each applied field. For visual clarity, the moment amplitude for $\mathrm{Yb} 2$ and $\mathrm{Yb} 3$ have been shifted slightly on the $x$ axis. The field values of the Yb1 moments correspond to the actual field values. Right scale: Black circles follow the field-dependent integrated intensity of the (110) nuclear peak.

the local moment directions and the exact positions of the magnetic phase boundaries.

Although the low-field phase diagram of $\mathrm{YbAgGe}$ retains all these features, there is still a significant deviation from this common trend. Usually, equal-moment collinear structures are favored at low temperatures. In YbAgGe, all magnetic structures, including the low-temperature zero-field one, are amplitude modulated. It is not clear to what extent this is dictated by geometrical frustration in the kagome layers or by the proximity to the non-Fermi-liquid state. However, it is apparent that the interplay of the CEF anisotropy of the three $\mathrm{Yb}$ sites, the applied magnetic field, and the frustrated exchange interactions play a significant role.

The enhanced moment amplitudes for Yb3 in the phases $c$

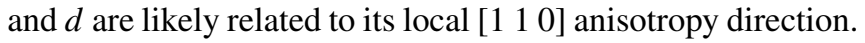
[ $\left.\begin{array}{lll}1 & 1 & 0\end{array}\right]$ is perpendicular to the applied-field direction and thus the field has no effect on the ordering of this sublattice. In contrast, the Yb1 and Yb2 magnetic sites are strongly influenced by the competition of the local CEF anisotropy and magnetic-field direction, such that they align either along their CEF axes (phases $a$ and $d$ ) or normal to them (phases $b$ and $c$ ). Remarkably, we observe significant $\hat{c}$ components in all proposed magnetic arrangements, which is hard to reconcile with the suggested $\mathrm{CEF}$ ground state of the $\mathrm{Yb}^{3+}$ ions [9]. Even so, in all these cases the in-plane components of the moments retain a 60 or 120 degrees canting with respect to the neighboring spins of the other two sublattices. Such a canted arrangement is reminiscent of the classical ground state of Heisenberg kagome antiferromagnets [23]. This implies that the interaction between the first neighbors in $\mathrm{YbAgGe}$ is antiferromagnetic and substantial.

A better understanding of the complex metamagnetic behavior in YbAgGe requires insight into Ruderman-KittelKasuya-Yosida (RKKY) exchange interactions of this material. This is challenging, as the Fermi surface of YbAgGe is anisotropic [2] and no reliable calculation of the electronic structure is available at this point. We attempt here a rough estimate of RKKY, simplifying the YbAgGe Fermi surface as being spherical and characterized by a single $k_{F}$ value, which we estimated from bulk measurements [18].

Given a carrier concentration of $n=3.1 \times 10^{21} \mathrm{~cm}^{-3}$, which has been normalized with the unit-cell volume and number of $\mathrm{Yb}$ ions, $n^{*}=n \times 178 \times 10^{-24} / 3$, we calculate

$$
k_{F}=\left(n^{*} \times 3 \pi^{2}\right)^{1 / 3}=1.76 \AA^{-1}
$$

Figure 7 presents the distance dependence of the RKKY interaction calculated as $\mathrm{J}\left(k_{F}\right)=C \times F\left(2 k_{F} r\right)$, where $C$ is a normalization constant and $F(x)=(\sin x-x \cos x) / x^{4}$. According to this model the RKKY exchange can be approximated by the bilinear AF in-plane exchange couplings up to fourth-nearest neighbor and a mixture of ferromagnetic and antiferromagnetic out-of-plane exchange couplings.

We note that this simplified picture has to be taken with caution. The propagation vectors identified for $\mathrm{YbAgGe}$ are not typical for the rest of the RAgGe family and are even more distant to other kagome or distorted-kagome compounds. The ordering vector $\mathbf{k}_{\mathbf{1}}=\left(\begin{array}{lll}\frac{1}{3} & 0 & \frac{1}{3}\end{array}\right)$ is very rarely observed in particular. We performed a Monte Carlo sampling of a Heisenberg Hamiltonian with up to twelve isotropic nearest-neighbor exchanges based on the generalized Luttinger-Tisza method [24] and detected only a few tiny pockets where the $\mathbf{k}_{\mathbf{1}}$ structure would be energetically stable. Thus presumably a more complex Hamiltonian that includes anisotropic exchanges, has to be considered.

Because the anomalous Hall effect was observed at the $c-d$ phase boundary [25], we inspected the topology of the determined magnetic structures by calculating the average scalar spin chirality,

$$
\chi=1 / N \sum_{j} \mathbf{S}_{1} \cdot\left(\mathbf{S}_{2} \times \mathbf{S}_{3}\right) .
$$

Here, the sum runs over all $j$ triangles formed by the Yb1, $\mathrm{Yb} 2$, and $\mathrm{Yb} 3$ moments in the magnetic unit cell. For the proposed 0-T (phase $a$ ), 2.2-T (phase $b$ ), and 5.5-T (phase $d$ ) models, the average chirality is zero within errors. In contrast, the two $\Gamma_{1} 3.9-\mathrm{T}$ solutions (phase $c$ ) reveal a finite average chirality of $0.3(1)$ and $-0.61(3)$, respectively. It is possible that the anomalous Hall effect in $\mathrm{YbAgGe}$ is induced by the spin chirality, similar to the frustrated-noncollinear-triangularlattice $\mathrm{Mn}_{3} \mathrm{Sn}$ [26] and $\mathrm{Fe}_{1.3} \mathrm{Sb}$ [27]. Thus other nontrivial properties such as topological band crossing and existence of 

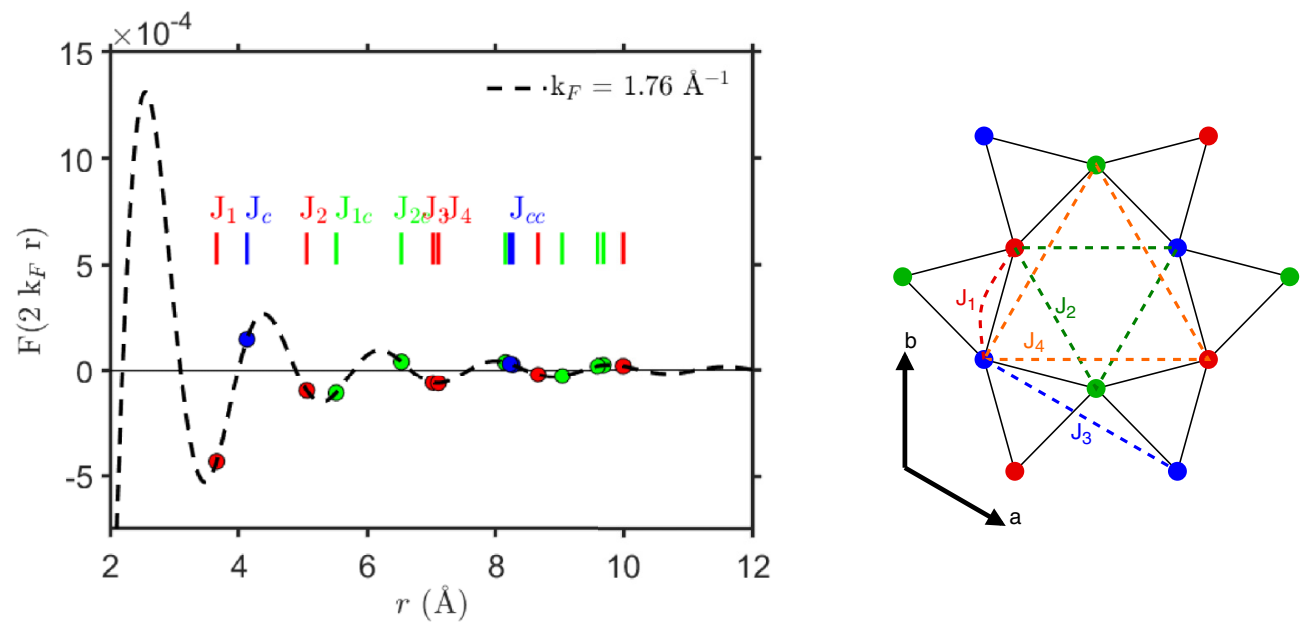

FIG. 7. An estimate of the RKKY exchange distance dependence (dashed line) plotted alongside markers for the Yb-Yb distances. $J_{1}-J_{4}$ are the in-plane nearest-neighbor exchanges, shown also in the right figure, $J_{c}, J_{c c}$ are exchanges in the $\hat{c}$ direction across one and two unit cells, respectively.

topological fermions may be expected in YbAgGe. Also, the scalar chirality may be driven by chiral fluctuations triggered by frustrated interactions, as is the case in the kagomecompound $\mathrm{YMn}_{6} \mathrm{Sn}_{6}$ [28].

The high-field features of the phase diagram (phases $c$ and $d$ ) merit further discussion. In phase $d$, the average moment amplitude of the Yb3 ion significantly exceeds (by almost four times) the values of the other two ions. This questions the role of the $\mathrm{Yb} 1$ and $\mathrm{Yb} 2$ local moments, i.e., whether they strongly fluctuate due to frustration, or whether their values are reduced due to the hybridization of $4 f$ and conduction electrons. To establish a site-specific variation of the hybridization for $\mathrm{Yb} 1 / \mathrm{Yb} 2$ and $\mathrm{Yb} 3$, these sites should become different under magnetic field. This could happen, if structural transition splitting the 3(f) site of $\mathrm{Yb}$ into two sites takes place. A possible hint of structural change is seen in the magnetostriction data [5], however no signature of an abrupt change in the crystal structure is observed in our neutron diffraction data. Thus, the dynamic coupling between the crystal lattice and the magnetic degrees of freedom has to be inspected carefully. A coupling between the CE levels and the phonons may be one possibility, which has been seen in $\mathrm{CeAuAl}_{3}$ for instance [29]. A strong coupling between CE levels and lattice vibrations would affect the elastic constants of YbAgGe, and has indeed been observed in previous reports [6]. Here, the softening of the elastic constants at $H_{\text {crit }}$ was attributed to the broadening of the $4 f$ level to gain Zeeman energy, which in turn yields a suppressed heavy-fermion behavior. To confirm such a scenario, state-of-the-art ab initio electronic-structure calculations, a study of the Fermi surface evolution when crossing the $\mathrm{BCP}$, or a verification of the magneto-elastic nature of the excitations are needed. Furthermore, numerous experimental results on $\mathrm{YbAgGe}$ show also evidence of non-Fermi-liquid behavior at the $c$ - $d$ phase boundary $[5,7]$, pointing to a scenario in which the $\mathrm{Yb} 1$ and $\mathrm{Yb} 2$ moments are strongly reduced due to critical fluctuations. Here theoretical models that help to understand how the $d$-phase magnetic structure and/or its associated excitation spectrum result in a linear $T$ dependence of the resistivity [3] are desired to make further progress in clarifying which of the aforementioned scenarios is dominant.

The tuning of the bicritical point at $T=0.3 \mathrm{~K}$ and $H=4.5 \mathrm{~T}$ to zero temperature is another exciting area of research. The application of pressure or chemical substitution, may push the BCP towards a quantum BCP, and further to a field-induced quantum spin-liquid state [7]. Actually, even in not-optimally tuned $\mathrm{YbAgGe}$, spin fluctuations may epitomize quantum spin-liquid-like character near $Q=\mathbf{k}_{\mathbf{3}}$. A study of the dynamic spin correlations using inelastic neutron scattering would be valuable to distinguish which of the two scenarios-quantum fluctuations of a metallic spin liquid state or a magnetic site-specific Kondo screening-is realized in YbAgGe.

Finally, the possibility of magnetic order being associated with the $e$ phase warrants further experimental investigation. The Fermi-liquid behavior of the $f$ phase could be consistent with fully field-polarized Yb moments, which is not associated with any finite propagation vector. However, the phase boundary between the $e$ and $f$ phase indicates a different magnetic order in the $e$ phase anticipating that a finite ordering vector could occur here. Therefore, it is salient to continue searching for the $e$-phase magnetic order either in the not-yetexplored part of reciprocal space or at $\mathbf{k}=(0,0,0)$.

\section{ACKNOWLEDGMENTS}

This work was performed at SINQ, Paul Scherrer Institute, Villigen, Switzerland with financial support of the Swiss National Science Foundation (Grant No. 200020-182536) and at ILL, Grenoble, France. Work at UCL was supported by the Engineering and Physical Sciences Research Council (EPSRC) under Grants No. EP/N027671/1 and EP/F032293/1. Work done at Ames Laboratory (PCC and SLB) was supported by the U.S. Department of Energy, Office of Basic Energy Science, Division of Materials Sciences and Engineering. Ames Laboratory is operated for the U.S. Department of Energy by Iowa State University under Contract No. DEAC02-07CH11358. PCC and SLB would like to acknowledge 
the long-standing interest and enthusiasm of George Schmiedeshoff in this project. We acknowledge A. S. Wills and J. Jensen for fruitful discussions and J. Schefer for help during the TriCS experiment.
[1] S. L. Bud'ko, E. Morosan, and P. C. Canfield, Phys. Rev. B 69, 014415 (2004).

[2] S. L. Bud'ko, V. Zapf, E. Morosan, and P. C. Canfield, Phys. Rev. B 72, 172413 (2005).

[3] P. G. Niklowitz, G. Knebel, J. Flouquet, S. L. Bud'ko, and P. C. Canfield, Phys. Rev. B 73, 125101 (2006).

[4] E. Mun, S. L. Bud'ko, and P. C. Canfield, Phys. Rev. B 82, 174403 (2010).

[5] G. M. Schmiedeshoff, E. D. Mun, A. W. Lounsbury, S. J. Tracy, E. C. Palm, S. T. Hannahs, J.-H. Park, T. P. Murphy, S. L. Bud'ko, and P. C. Canfield, Phys. Rev. B 83, 180408(R) (2011).

[6] Y. Nakanishi, R. Kashiwazaki, K. Deto, F. Shichinomiya, M. Nakamura, H. Kubo, K. Umeo, T. Onimaru, T. Takabatake, and M. Yoshizawa, J. Phys.: Conf. Ser. 273, 012011 (2011).

[7] Y. Tokiwa, M. Garst, P. Gegenwart, S. L. Bud'ko, and P. C. Canfield, Phys. Rev. Lett. 111, 116401 (2013).

[8] B. Fåk, D. F. McMorrow, P. G. Niklowitz, S. Raymond, E. Ressouche, J. Flouquet, P. C. Canfield, S. L. Bud'ko, Y. Janssen, and M. J. Gutmann, J. Phys.: Condens. Matter 17, 301 (2005).

[9] T. Matsumura, H. Ishida, T. J. Sato, K. Katoh, Y. Niide, and A. Ochiai, J. Phys. Soc. Jpn. 73, 2967 (2004).

[10] B. Fåk, Ch. Rüegg, P. G. Niklowitz, D. F. McMorrow, P. C. Canfield, S. L. Bud'ko, Y. Janssen, and K. Habicht, Phys. B: Condens. Matter 378-380, 669 (2006).

[11] P. Bonville, M. Rams, K. Królas, P. C. Canfield, J.-P. Sanchez, O. Trovarelli, and C. Geibel, Eur. Phys. J. B 55, 77 (2007).

[12] H. Kubo, K. Umeo, K. Katoh, A. Ochiai, and T. Takabatake, J. Phys. Soc. Jpn. 79, 064715 (2010).

[13] G. Zanicchi, D. Mazzone, V. Contardi, R. Marazza, G. Rambaldi, and D. Rossi, Gazz. Chim. Ital. 113, 257 (1983).
[14] E. Morosan, S. L. Bud'ko, P. C. Canfield, M. S. Torikachvili, and A. H. Lacerda, J. Magn. Magn. Mater. 277, 298 (2004).

[15] E. Morosan, S. L. Bud'ko, and P. C. Canfield, Phys. Rev. B 71, 014445 (2005).

[16] K. Zhao, H. Deng, H. Chen, K. A. Ross, V. Petříček, G. Günther, M. Russina, V. Hutanu, and P. Gegenwart, Science 367, 1218 (2020).

[17] K. Katoh, Y. Mano, K. Nakano, G. Terui, Y. Niide, and A. Ochiai, J. Magn. Magn. Mater. 268, 212 (2004).

[18] S. L. Bud'ko, E. Morosan, and P. C. Canfield, Phys. Rev. B 71, 054408 (2005).

[19] Y. Tokiwa, A. Pikul, P. Gegenwart, F. Steglich, S. L. Bud'ko, and P. C. Canfield, Phys. Rev. B 73, 094435 (2006).

[20] P. C. Canfield, Rep. Prog. Phys. 83, 016501 (2019).

[21] J. Rodríguez-Carvajal, Phys. B: Condens. Matter 192, 55 (1993).

[22] D. Gignoux and D. Schmitt, Phys. Rev. B 48, 12682 (1993).

[23] J. T. Chalker, P. C. W. Holdsworth, and E. F. Shender, Phys. Rev. Lett. 68, 855 (1992).

[24] J. M. Luttinger and L. Tisza, Phys. Rev. 70, 954 (1946).

[25] H. Ishizuka and N. Nagaosa, Sci. Adv. 4, eaap9962 (2018).

[26] S. Nakatsuji, N. Kiyohara, and T. Higo, Nature (London) 527, 212 (2015).

[27] Y. Shiomi, M. Mochizuki, Y. Kaneko, and Y. Tokura, Phys. Rev. Lett. 108, 056601 (2012).

[28] N. J. Ghimire, R. L. Dally, L. Poudel, D. C. Jones, D. Michel, N. T. Magar, M. Bleuel, M. A. McGuire, J. S. Jiang, J. F. Mitchell, J. W. Lynn, and I. I. Mazin, Sci. Adv. 6, eabe2680 (2020).

[29] P. Čermák, A. Schneidewind, B. Liu, M. M. Koza, C. Franz, R. Schönmann, O. Sobolev, and C. Pfleiderer, Proc. Natl. Acad. Sci. USA 116, 6695 (2019). 